'Servicio de Medicina Interna, Hospital Carlos Van Buren. Valparaíso, Chile.

2Departamento de Medicina, Escuela de Medicina, Universidad de Valparaíso. Viña del Mar, Chile.

${ }^{3}$ Servicio de Medicina, Sección Nefrología, Hospital Naval A. Nef. Viña del Mar, Chile.

${ }^{4}$ Servicio de Medicina, Sección Nefrología, Hospital Dr. Gustavo Fricke. Viña del Mar, Chile.

Recibido el 2 de marzo de 2017, aceptado el 19 de junio de 2017.

Correspondencia a:

Dr. Alejandro Kral

6 Oriente 230, Depto 72, Viña del Mar, Chile. alejandro.kral@gmail.com

\section{Síndrome de McKittrick-Wheelock. Una causa infrecuente de hipokalemia e injuria renal aguda. Caso clínico}

\author{
ALEJANDRO KRAL ${ }^{1,2}$, JORGE VEGA ${ }^{2,3,4}$ \\ McKrittick-Wheelock syndrome.
Report of one case
}

McKittrick-Wheelock syndrome is caused by chronic water and electrolyte hypersecretion from an intestinal tumor, usually a villous adenoma, located in the rectum or sigmoid. Patients often have dehydration, hypovolemic shock and kidney failure associated with hypokalemia, hyponatremia, hypochloremia and metabolic acidosis. We report a 62-year-old male, suffering chronic diarrhea for eight years who was admitted after a syncope. He had severe hypokalemia, hyponatremia, metabolic acidosis, hypovolemia and acute renal failure. After his metabolic disorders were corrected, a colonoscopy showed a large rectosigmoid tumor with the characteristics of a villous adenoma. During the follow up after the complete tumor resection, the patient has remained asymptomatic.

(Rev Med Chile 2017; 145: 950-953)

Key words: Acidosis; Adenoma, Villous; Diarrhea; Hypokalemia; Renal Insufficency.

\section{L} a colonoscopía es un examen endoscópico frecuentemente realizado, tanto en situaciones en que se sospecha patología colónica, como también para pesquisar cáncer de colon y recto $^{1}$. En $30 \%$ de los pacientes en que se efectúa el procedimiento con este fin, se encuentran uno o varios adenomas, a menudo de pocos milímetros de diámetro, que suelen ser asintomáticos ${ }^{2}$. De acuerdo a su estructura histológica se clasifican en adenomas tubulares, túbulo-vellosos y vellosos ${ }^{2}$, los cuales tienen asociado un potencial de malignidad según el tamaño de estos. En 2 a $3 \%$ de los adenomas vellosos, habitualmente de varios centímetros, localizados en recto o recto-sigmoides, se produce hipersecreción de un contenido mucoso rico en electrolitos pudiendo ocasionar el síndrome de McKittrick-Wheelock $(\mathrm{SMKW})^{2}$. Este consiste en una diarrea crónica de alto volumen (a veces sobre 4 litros diarios) que lleva a deshidratación, hipokalemia marcada, hiponatremia, hipocloremia, acidosis metabólica e injuria renal aguda, $y$ frecuentemente puede pro- ducir shock hipovolémico e hipoproteinemia ${ }^{1,3}$. A este trastorno también se lo ha llamado "cólera neoplásico" ${ }^{4}$. De no ser tratado, su mortalidad es de $100 \%{ }^{5}$.

El SMKW es una patología rara, existiendo alrededor de 50 casos publicados según registros de Pubmed hasta fines de $2016^{3}$. En Chile se han publicado dos casos en Revista Médica de Chile ${ }^{6,7}$.

Comunicamos el caso de un paciente que ingresó al Hospital Carlos Van Buren por sintomatología cardiovascular sugerente de enfermedad coronaria y que durante su hospitalización se diagnosticó este raro síndrome.

\section{Caso clínico}

Hombre de 62 años, campesino, sin antecedentes mórbidos. Ingresó al hospital después de una lipotimia con alteraciones electrocardiográficas sugerentes de isquemia miocárdica (Figura 1), lo que se descartó ulteriormente. Estaba muy 
enflaquecido, refiriendo, además, un cuadro de diarrea acuosa desde hacía 8 años. Al ingreso existía hipokalemia severa $(1,7 \mathrm{mEq} / \mathrm{L})$, hiponatremia $(131 \mathrm{mEq} / \mathrm{L})$ y disfunción renal (nitrógeno ureico $81 \mathrm{mg} / \mathrm{dl}$, creatinina 1,68 mg/dl). El hemograma, calcemia, fosfemia y albuminemia eran normales.

Se trató con hidratación parenteral y aporte de potasio durante la primera semana, logrando obtener concentraciones de potasio superiores a 3,5 $\mathrm{mEq} / \mathrm{L}$, persistiendo la diarrea (más de 3 episodios diarios). $\mathrm{Al} 6^{\circ}$ día de evolución presentó hipotensión arterial, oliguria y acentuación de la disfunción renal (nitrógeno ureico $138 \mathrm{mg} / \mathrm{dl}$, creatinina $8,0 \mathrm{mg} / \mathrm{dl})$, de la hiponatremia $(120 \mathrm{mEq} / \mathrm{L})$ y de la acidosis metabólica (bicarbonato $8,3 \mathrm{mEq} / \mathrm{L}$ ), requiriendo de una sesión de hemodiálisis.

Al tacto rectal había un esfínter hipertónico y el recto estaba ocupado por una masa renitente que

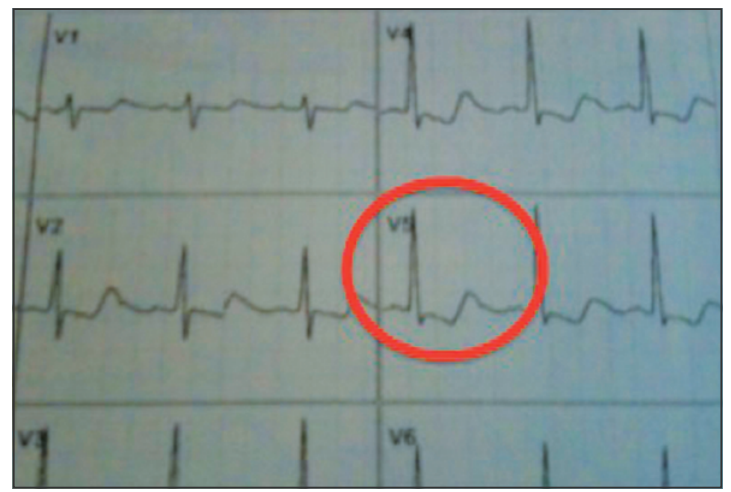

Figura 1. Electrocardiograma de ingreso. Se observa en las derivaciones precordiales infradesnivel del segmento S-T, que fue inicialmente interpretado como secundario a isquemia miocárdica. comprometía todo su perímetro. La ecotomografía abdominal y pelviana describió una ampolla rectal de $7 \mathrm{~cm}$ de diámetro transversal con material sólido en su interior.

Durante la segunda y tercera semana de hospitalización, la función renal mejoró en forma significativa (creatinina $2,56 \mathrm{mg} / \mathrm{dl}$ ), siempre bajo la administración de soluciones de hidratación parenteral y aportes de potasio. La colonoscopía (Figura 2) mostró una extensa lesión plana con aspecto de adenoma entre los 5 y $20 \mathrm{~cm}$ del margen anal. La biopsia rectal reveló un adenoma velloso con displasia epitelial severa.

Se planteó el diagnóstico de síndrome de McKittrick-Wheelock, decidiéndose la resección del tumor. Al día $49^{\circ}$ de la hospitalización se efectuó resección anterior del recto con anastomosis término-terminal. La biopsia de la pieza operatoria (Figura 2) fue informada como adenoma velloso de la mucosa rectal con microfocos de adenocarcinoma in situ. Los márgenes de resección estaban libres de tumor y los ganglios linfáticos no contenían metástasis.

El paciente evolucionó sin complicaciones en el postoperatorio y egresó sin diarrea y con normalidad de su función renal y trastornos metabólicos. En los controles ambulatorios posteriores estaba asintomático.

\section{Discusión}

Este paciente ingresó al hospital después de una lipotimia y tuvo alteraciones electrocardiográficas que hicieron pensar en una patología coronaria. Estas alteraciones fueron manifestación de la

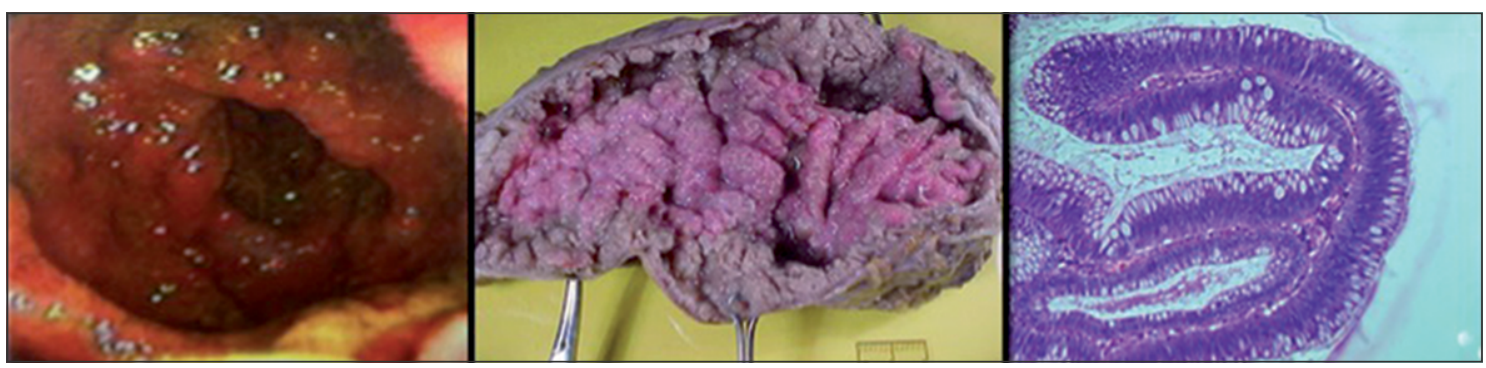

Figura 2. Imágenes de la colonoscopía, pieza resecada y su histología. Se observa lesión tumoral que compromete todo el perímetro del recto. La histología mostró un adenoma con presencia abundante de vellosidades digitiformes con abundantes células caliciformes en las células intestinales. 
hipovolemia e hipokalemia severa $(1,7 \mathrm{mEq} / \mathrm{L})$ secundaria a la diarrea secretora que sufría desde hacía 8 años. En los casos publicados, la duración de la diarrea a menudo ha sido prolongada, variando entre 1 mes y 10 años (promedio 23 meses) ${ }^{1}$.

Los enterocitos normales reabsorben agua, sodio y bicarbonato y secretan potasio ${ }^{8}$. Las células intestinales del adenoma velloso hipersecretor tienen un intercambiador de bicarbonato en su superficie apical inapropiadamente activado, llevando a depleción de bicarbonato, además de potasio, sodio y agua ${ }^{4}$. Este paciente presentó acidosis metabólica severa secundaria a la pérdida intestinal de bicarbonato y a la injuria renal aguda con características prerrenales secundaria a la hipovolemia. En algunas ocasiones, cuando la función renal no está comprometida, puede existir alcalosis metabólica originada por hiperaldosteronismo secundario por la pérdida de volumen y potasio por las heces ${ }^{3}$. En los casos publicados, los valores plasmáticos de potasio y sodio promedio fueron de 2,57 y $118 \mathrm{mEq} / \mathrm{L}$, respectivamente ${ }^{1}$. En las deposiciones de los pacientes con SMKW, las concentraciones promedio de sodio, potasio y cloro fueron $120,4,4$ y $123 \mathrm{mEq} / \mathrm{L}$, respectivamente ${ }^{2}$.

Las prostaglandinas (PGE2) producidas por las células del adenoma velloso se han implicado como el secretagogo responsable de la diarrea secretora ${ }^{9}$. La PGE2 provocaría un aumento del AMP cíclico intracelular, el que activaría los canales apicales de cloro y potasio de las células epiteliales, provocando la salida masiva de agua y electrolitos. En las deposiciones se han encontrado niveles elevados de PGE2 y en la histología expresión intensa de la COX-2 en las glándulas del tumor ${ }^{1,3}$. Cuando el tumor ha sido resecado, las concentraciones de PGE2 en las heces se normalizan?.

La injuria renal aguda es uno de los componentes del síndrome. La causa de ella es la isquemia renal producida por hipovolemia originada por las pérdidas digestivas. Este paciente ingresó con una injuria renal típicamente del tipo prerrenal (uremia $173 \mathrm{mg} / \mathrm{dl}$, creatinina 1,68 mg/dl, relación BUN/creatinina 48,2), la que durante la primera semana de hospitalización se agravó, requiriendo de una sesión de hemodiálisis. Afortunadamente, como en la mayoría de los casos publicados, la falla renal revirtió con la reposición de volumen y electrolitos.

Los adenomas vellosos productores del SMKW habitualmente son mayores de $3 \mathrm{~cm}$ de diámetro, pudiendo alcanzar hasta $18 \mathrm{~cm}$ de longitud ${ }^{3}$. La mayoría son sésiles y están localizados en el recto, donde la capacidad de reabsorción del agua secretada bajo el tumor es nula ${ }^{1}$. Por tanto, la localización, así como el tamaño del tumor, son factores que determinan la intensidad de la sintomatología de los pacientes.

En la histología de los adenomas vellosos es característica la presencia de prolongaciones digitiformes compuestas por epitelio intestinal con abundante contenido de mucina en la porción apical de las células ${ }^{3}$. La presencia de displasia epitelial severa es frecuente en los adenomas vellosos y alrededor de $80 \%$ tienen focos microscópicos de adenocarcinoma, como sucedió en este paciente ${ }^{9}$. Los adenomas vellosos menores de $1 \mathrm{~cm}$ tienen un riesgo de transformarse en carcinomas invasores en más de 10 veces respecto a los adenomas tubulares. Cuando su diámetro es mayor de $2 \mathrm{~cm}$ el riesgo de malignizarse es de $50 \%{ }^{2}$.

El SMKW ocasionalmente se ha originado en adenocarcinomas y tumores neuroendocrinos ${ }^{1}$. Si bien la diarrea frecuentemente es acuosa o mucosa, ocasionalmente puede acompañarse de sangre, por ulceración del tumor ${ }^{1}$. Se han comunicado casos en que se ha producido invaginación o prolapso rectal $^{1,5}$. Muy rara vez el adenoma velloso puede asociarse a diabetes mellitus, la que desaparece después de la extirpación del tumor; acompañarse de infección por Clostridium difficile o coexistir junto a un síndrome de Cronkhite-Canada ${ }^{5,10,11,13}$.

El tratamiento inmediato del SMKW consiste en la hidratación y reposición de electrolitos, para corregir la hipovolemia y alteraciones metabólicas; y posteriormente la resección del tumor. Para esto último se ha empleado la cirugía abierta, laparoscópica y endoscópica, así como la radioterapia endocavitaria, la que se asocia a una mayor tasa de recurrencia ${ }^{2,3}$. En los pacientes que no aceptan o toleran la cirugía, se ha empleado con éxito variable el uso de antiinflamatorios no esteroidales (mayoritariamente indometacina), inhibidores de la COX-2 y análogos de la somatostatina ${ }^{3,9}$. El uso de estas drogas hace que el volumen de las deposiciones y la concentración de sodio disminuyan, así como la concentración de PGE2 ${ }^{3}$.

En este paciente, la resección del tumor hizo desaparecer definitivamente la diarrea y las alteraciones metabólicas asociadas y permitió detener la evolución de su tumor a un adenocarcinoma 
rectal invasor, dados los focos microscópicos de adenocarcinoma encontrados en la pieza resecada. Creemos que este caso, en que para el diagnóstico se utilizó la clínica y medios de apoyo básicos disponibles en todos los hospitales, ilustra la sospecha de este raro síndrome en pacientes que consultan a los servicios de urgencia por falla renal aguda e hipokalemia asociado a antecedentes de diarrea crónica.

\section{Referencias}

1. Malik S, Mallick B, Makkar K, Kumar V, Sharma V, Rana SS. Malignant McKittrick-Wheelock syndrome as a cause of acute kidney injury and hypokalemia: report of a case and review of literatura. Intractable Rare Dis Res 2016; 5 (3): 218-21.

2. Raphael MJ, McDonald CM, Detsky AS. McKittrick-Wheelock syndrome. CMAG 2015; 187 (9): 676-8.

3. Tuta LA, Bosoteanu M, Deacu M, Dumitru E. McKittrick-Wheelock síndrome: a rare etiology of acute renal failure associated to well-differentiated adenocarcinoma (G1) arising within a villous adenoma. Rom J Morphol Embryol 2011; 52 (3 Suppl); 1153-6.

4. Podestà MA, Cucchiari D, Merizzoli E, Elmore U, Angelini C, Badalamenti S. McKittrick-Wheelock syndrome: a rare cause of acute renal failure and hypokalemia not to be overlooked. Ren Fail 2014; 36 (5): 811-3.

5. Mois E, Graur F, Sechel R, Al-Hajjar N. McKit-
trick-Wheelock Syndrome: a rare case report of acute renal failure. Clujul Med 2016; 89 (2): 301-3.

6. Suárez J. Síndrome de McKittrick y Wheelock. Caso clínico. Rev Med Chile 1994; 122 (2): 198-200.

7. Mila R, Grille S, Laurini M, Lapiedra D, Bagattini JC. Síndrome de McKittrick-Wheelock: Una causa infrecuente de shock hipovolémico. Rev Med Chile 2008; 136: 900-4.

8. Choi WH, Ryuk J, Kim HJ, Park SY, Park JS, Kim JG, et al. A case of giant rectal villous tumor with severe fluid-electrolyte imbalance treated by laparoscopic low anterior resection. J Korean Surg Soc 2012; 82: 325-9.

9. Agnes A, Novelli D, Doglietto GB, Papa V. A case report of a giant rectal adenoma causing secretory diarrea and acute renal failure: McKrittick-Wheelock syndrome. BMC Surgery 2016; 16: 39.

10. Challis BG, Lim CT, Cluroe A, Cameron E, O’Rahilly S. The McKittrick-Wheelock syndrome: a rare cause of curable diabetes. Endocrinology, Diabetes \& Metabolism. Case reports 2016; 1-6.

11. Watari J, Sakurai J, Morita T, Yamasaki T, Okugawa T, Toyoshima F, et al. A case of Cronkhite-Canada syndrome complicated by McKittrick-Wheelock syndrome associated with advanced villous adenocarcinoma. Gastrointest Endosc 2011; 73 (3): 624-6.

12. Learney RM, Ziprin P, Swift PA, Faiz OD. Acute renal failure in association with community-adquired Clostridium difficile infection and McKittrick-Wheelock syndrome. Case Rep Gastroenterol 2011; 5 (2): 438-44. 